\title{
The risks of extreme load extrapolation
}

\author{
Stefan F. van Eijk, René Bos, and Wim A. A. M. Bierbooms \\ Wind Energy Research Group, Faculty of Aerospace Engineering, Delft University of Technology, \\ 2629 HS Delft, the Netherlands \\ Correspondence to: Stefan F. van Eijk (sfvaneijk@gmail.com)
}

Received: 2 March 2017 - Discussion started: 14 March 2017

Revised: 25 May 2017 - Accepted: 19 June 2017 - Published: 14 July 2017

\begin{abstract}
An important problem in wind turbine design is the prediction of the 50-year load, as set by the IEC 61400-1 Design Load Case (DLC) 1.1. In most cases, designers work with limited simulation budgets and are forced to use extrapolation schemes to obtain the required return level. That this is no easy task is proven by the many studies dedicated to finding the best distribution and fitting method to capture the extreme load behavior as well as possible. However, the issue that is often overlooked is the effect that the sheer uncertainty around the 50-year load has on a design process. In this paper, we use a collection of 96 years' worth of extreme loads to perform a large number of hypothetical design problems. The results show that, even with sample sizes exceeding $N=10^{3} 10 \mathrm{~min}$ extremes, designs are often falsely rejected or falsely accepted based on an overor underpredicted 50-year load. Therefore, designers are advised to be critical of the outcome of DLC 1.1 and should be prepared to invest in large sample sizes.
\end{abstract}

\section{Introduction}

Wind turbine designers are confronted with the IEC 61400-1 Design Load Case (DLC) 1.1 (IEC, 2005). This evaluates the structural integrity of the major load-carrying components on the basis of a 50-year return level, plus safety factors. As prescribed by Appendix $\mathrm{F}$ of the standards, a minimum of $300 \mathrm{~min}$ of time series - distributed over the relevant wind speeds - will have to be evaluated and followed by an extrapolation scheme to obtain the 50-year return level. Such extrapolations produce notoriously uncertain estimates, which is why the Design Load Case 1.1 is often avoided or at least greatly simplified in early stages of the design. However, in cases where DLC 1.1 is design-driving (e.g., for foundations and controllers), dealing with this uncertainty is unavoidable.

Many past efforts to reduce this uncertainty have focused on trying out different sampling methods (Fogle et al., 2008; Agarwal and Manuel, 2009), new modeling techniques (Moriarty et al., 2004; Bos and Veldkamp, 2016), or finding the best distribution type to match the extreme load behavior (Pandey and Sutherland, 2003; Genz et al., 2006; Freudenreich and Argyriadis, 2007; Ragan and Manuel, 2007; Natarajan and Holley, 2008; Peeringa, 2009; Lott and Cheng,
2016). Yet, because most studies deal with relatively small sample sizes (e.g., $\ll 1$ year), the actual uncertainty that surrounds the 50-year return level is often underexposed. With a 63-year data set, Barone et al. (2012b) were able to establish the $90 \%$ confidence interval around the 50-year load for sample sizes up to $N=200010 \mathrm{~min}$ maxima. This revealed not only that the 50 -year levels are clouded by high uncertainty but also that they suffer from a considerable bias. Inevitably, this has an effect on the choices made during design.

The aim of this paper is to demonstrate this with a simple exercise, using a collection of 96 years' worth of $10 \mathrm{~min}$ load maxima released by Barone et al. (2012a). The uncertainty distribution is constructed by repeatedly sampling subsets of this data set and obtaining the 50-year loads through an automated extrapolation scheme. We then simulate a problem where a hypothetical designer has to choose between two or more concepts and record how often this uncertainty leads to wrong choices. The results of this paper should help designers to estimate the required sample sizes for their problem but also to form a critical attitude concerning the quality and reliability of extrapolated 50-year loads. 


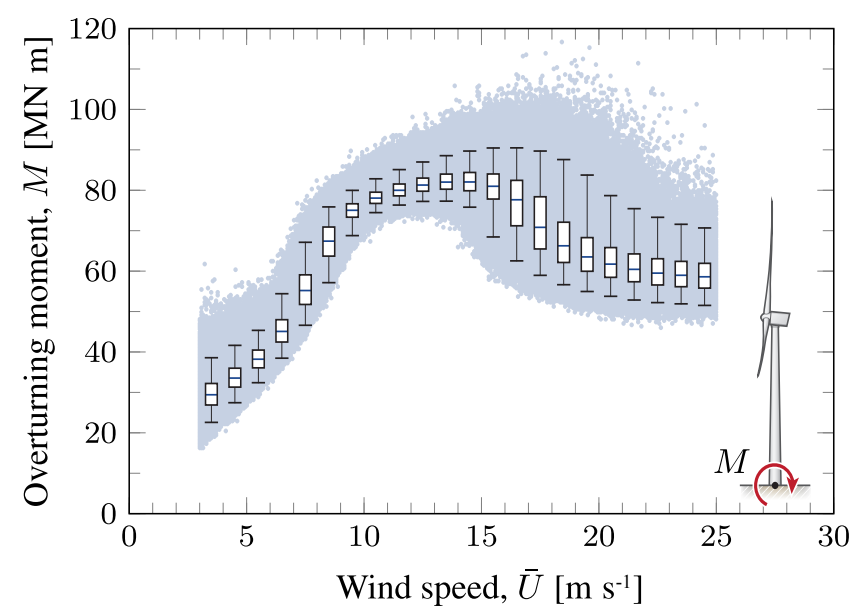

Figure 1. The data set, containing over 5 million 10 min extreme overturning moments between the cut-in and cut-out wind speeds. The box plots indicate the scatter per $1 \mathrm{~m} \mathrm{~s}^{-1}$ bin, where the boxes mark the 25 th and 75 th percentiles, the whiskers mark the 2.5 th and 97.5th percentiles, and the bar is the median.

\section{Methodology}

Since the focus of this work is on the impact of uncertainty, rather than on obtaining the highest possible quality result, the workflow is kept as simple as possible. Loads were extracted by drawing a random sample from a large set of crude Monte Carlo results and the 50-year return period is found by a graphical fit.

\subsection{Loads data set}

The data set that was used for this study was generated by Barone et al. (2012a). It features the onshore version of the NREL $5 \mathrm{MW}$ reference wind turbine, operating for 96 years in an IEC class 1B climate (IEC, 2005). ${ }^{1}$ Ten-minute mean wind speeds were randomly drawn from a Rayleigh distribution, bounded by the cut-in and cut-out wind speeds of 3 and $25 \mathrm{~m} \mathrm{~s}^{-1}$, respectively. Turbulent wind fields were generated by TurbSim on a $20 \times 20$ grid with a width and height of $137 \mathrm{~m}$ and were fed to the FAST v7 aeroelastic code. Every simulation ran for $11 \mathrm{~min}$, of which the first minute was discarded to avoid any start-up transients. More details can be found in the original paper.

Each output channel contains over 5 million 10 min extremes. In this paper, we will use the tower base overturning moment, which plays a major role in the design of foundations. Figure 1 shows the entire set of loads at the respective wind speeds.

\footnotetext{
${ }^{1}$ The original paper specifies a class $2 \mathrm{~B}$ site, but this has been corrected with the release of the data set (see http://energy.sandia. gov/?page_id=13173).
}

\subsection{Extrapolation scheme}

In many practical situations, a designer does not have the computational resources available to simulate several decades of operation. That is when the 50-year load has to be found by extrapolating.

\subsubsection{Aggregation before fitting and fitting before aggregation}

There are several approaches to the extrapolation problem. One method involves drawing a sample directly from the parent mean wind speed distribution. The cumulative distribution of extreme loads then follows naturally from ranking a set of $N$ loads and assigning a plotting position:

$$
\hat{F}\left(M_{i}\right)=\frac{i}{N+1} .
$$

In this case, however, the wind speeds outside of the operating range will have to be accounted for:

$$
\hat{F}\left(M_{i}\right)=1-\left(1-\frac{i}{N+1}\right) \int_{\bar{U}_{\text {cut-in }}}^{\bar{U}_{\text {cut-out }}} f(\bar{U}) \mathrm{d} \bar{U}
$$

where $f(\bar{U})$ is the mean wind speed distribution. Then, plotting the entire data set yields the return level plot shown in Fig. 2. Extrapolation is done by using the entire sample, called aggregation before fitting. With 96 years' worth of load data, however, the 50-year return value can be easily matched with a generalized extreme value (GEV) distribution (see next subsection), which yields $115.0 \mathrm{MN} \times \mathrm{m}$. Repeating the process by randomly drawing 96 -year samples from the same data set allows us to estimate the $95 \%$ confidence interval, yielding $[113.1,117.2] \mathrm{MN} \times \mathrm{m}$.

Sampling directly from the parent distribution is an example of a crude Monte Carlo method, which has the advantage that it gives a raw and unbiased picture of the extreme loads. However, a clear disadvantage is that the bulk of the data originates from relatively low wind speeds where the extremes loads are not expected to lie. In addition, unless a stratified sampling method is used, the wind speeds in any small subsample are not always representative of the parent distribution.

Another method, which is preferred by the IEC guidelines (IEC, 2005), requires the data to be collected in $n$ wind speed bins of a certain width, $\Delta \bar{U}$. Data from every bin is then matched with a distribution function, after which every dis- 


\section{Return period, $T$}

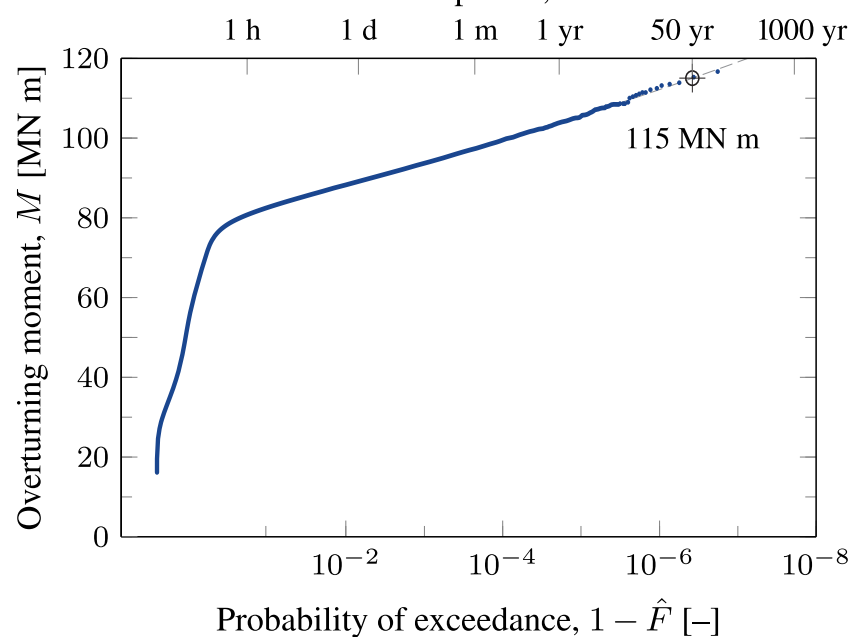

Figure 2. Return level plot of the tower base overturning moment, with the entire 96-year data set (i.e., aggregation before fitting). A GEV fit above the threshold given by Eq. (6) yields a 50-year value of $115.0 \mathrm{MN} \times \mathrm{m}$.

tribution is weighted according to

$$
\begin{aligned}
\hat{F}(M)= & 1-\int_{\bar{U}_{\text {cut-in }}}^{\bar{U}_{\text {cut-out }}} f(\bar{U}) \mathrm{d} \bar{U}+\sum_{i=1}^{n} F\left(M \mid \bar{U}_{i}\right) \\
& \int_{\bar{U}_{i}+\frac{1}{2} \Delta \bar{U}} f(\bar{U}) \mathrm{d} \bar{U} .
\end{aligned}
$$

This is called fitting before aggregation. It has the advantage that the data in each wind speed bin have less variation and match an underlying distribution more closely. However, the obvious disadvantage is that a factor $n$ fewer data points are available in each bin to fit (see, e.g., Figs. 3 and 4).

\subsubsection{Choice of distribution function}

The tail behavior is matched with a distribution function, for example by least-squares fitting. A good candidate for this is the generalized extreme value distribution (GEV):

$G(M ; \mu, \sigma, \xi)=\exp \left[-\left(1+\xi \frac{M-\mu}{\sigma}\right)^{-1 / \xi}\right]$,

where $\mu$ is the location parameter, $\sigma$ the scale parameter, and $\xi$ the shape parameter. A possible alternative is to fix $\xi=0$, which produces a two-parameter Gumbel distribution:

$G(M ; \mu, \sigma)=\exp \left[-\exp \left(-\frac{M-\mu}{\sigma}\right)\right]$.

A Gumbel distribution appears as a perfectly straight line on Gumbel paper (i.e., on a double-logarithmic scale as in Fig. 2) and is often a good first guess of the tail behavior.

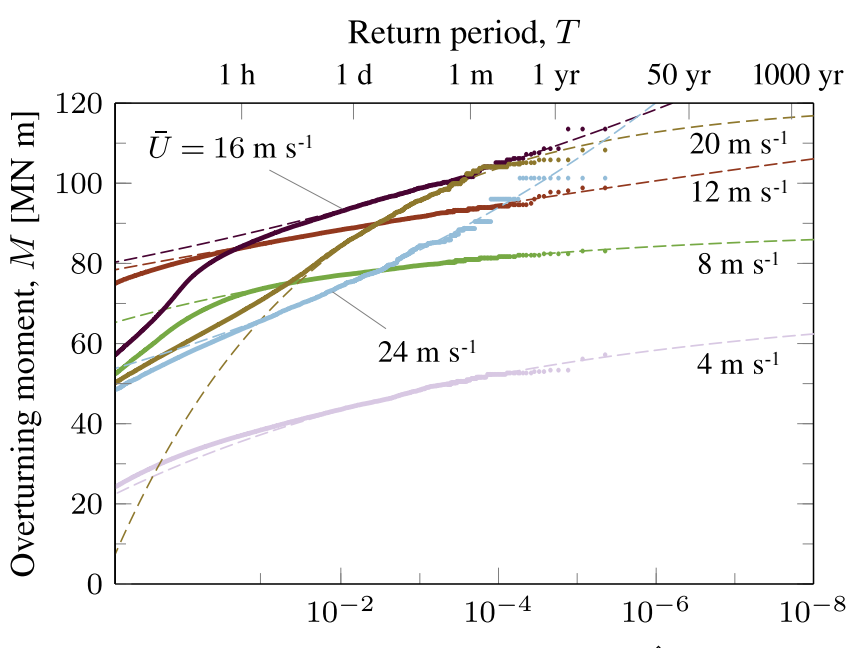

Probability of exceedance, $1-\hat{F}[-]$

Figure 3. Return level plot of some $1 \mathrm{~m} \mathrm{~s}^{-1}$ wide bins with a GEV distribution fit after a threshold given by Eq. (6).

Return period, $T$

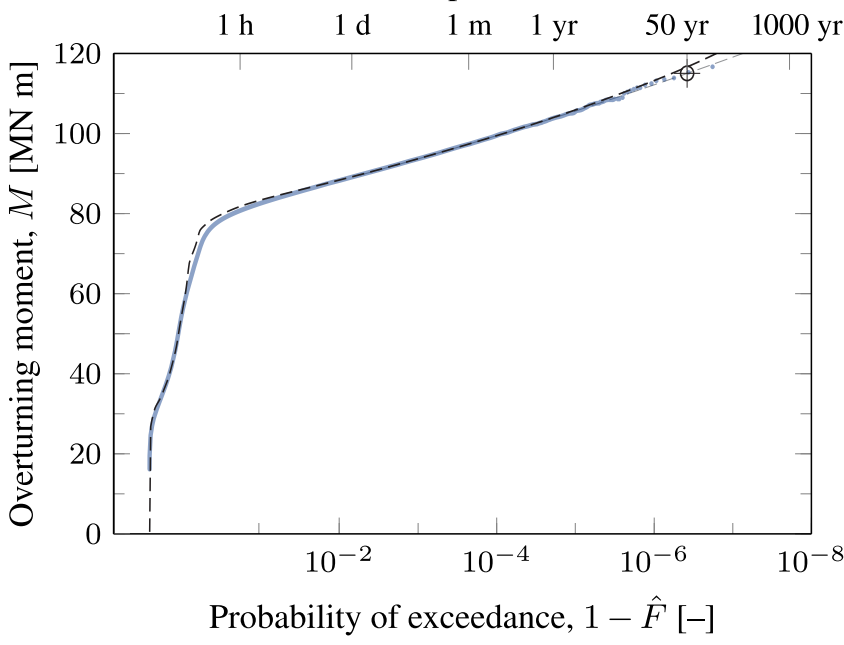

Figure 4. Return level plot of the tower base overturning moment by a weighted sum of the bins shown in Fig. 3 (i.e., fitting before aggregation), using an equivalent 96-year sample size. The weighted distribution is given by a black dashed line and is overlaid on Fig. 2 for comparison.

\subsubsection{Identifying the distribution tail}

The tail of the distribution shows a characteristic bend, or "knee", that indicates that more than one process is at work. Indeed, tracing back the wind speeds belonging to the $10 \%$ highest loads points towards a region well above the rated wind speed (see Fig. 5). It turns out that this is due to a particular controller response to negative gust amplitudes (e.g., Bos et al., 2015; Bos and Veldkamp, 2016), which also explains the shape of the scatterplot in Fig. 1. 


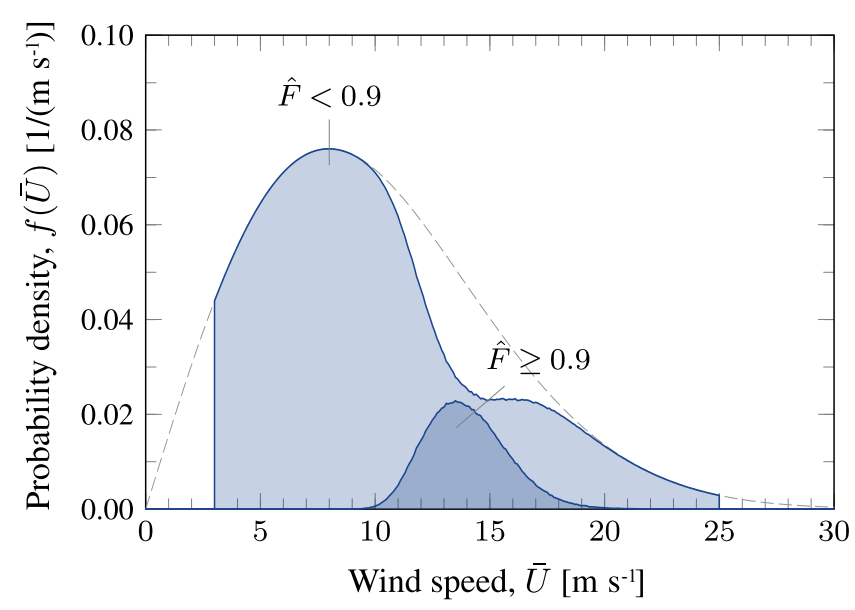

Figure 5. Histogram of the sampled wind speeds, where the dashed line marks the Rayleigh mean wind speed distribution belonging to an IEC class $1 \mathrm{~B}$ climate. The light and dark filled areas correspond to the lowest $90 \%$ and highest $10 \%$ loads, respectively.

To estimate the uncertainty that comes from repeatedly extrapolating different sets of loads, this process has to be automated. However, the difficult part is then to decide exactly where the tail starts under varying sample size. A simple solution that seems to work in most cases is to assume that the tail covers the second half of the distribution when drawn on Gumbel paper, i.e., above a threshold

$$
\begin{aligned}
-\ln [-\ln [\hat{F}]]> & -\frac{1}{2} \ln \left[-\ln \left[\hat{F}\left(M_{1}\right)\right]\right] \\
& -\frac{1}{2} \ln \left[-\ln \left[\hat{F}\left(M_{N}\right)\right]\right] .
\end{aligned}
$$

For the full data set, this means that a distribution is fitted to the upper $0.07 \%$ of the data. For a GEV fit, this results in the quantile-quantile (Q-Q) plot shown in Fig. 6.

\subsection{Workflow}

The different approaches discussed in the previous subsection are used to set up eight extrapolation cases (see Table 1). The sampling method describes whether the data are drawn directly from their parent Rayleigh distribution and fitted to the entire set (aggregation before fitting) or if they are constructed from $1 \mathrm{~m} \mathrm{~s}^{-1}$ wide bins with an equal number of data points per bin (fitting before aggregation). Extrapolation is done by matching the data points with either a Gumbel or GEV distribution by least-squares fit. The final column states whether the entire sample is used (No) or only the points that lie above the threshold given by Eq. (6) (Yes).

These cases are automated for $k=10^{3}$ sets of loads, which yields a collection of 50-year return levels, distributed ac-

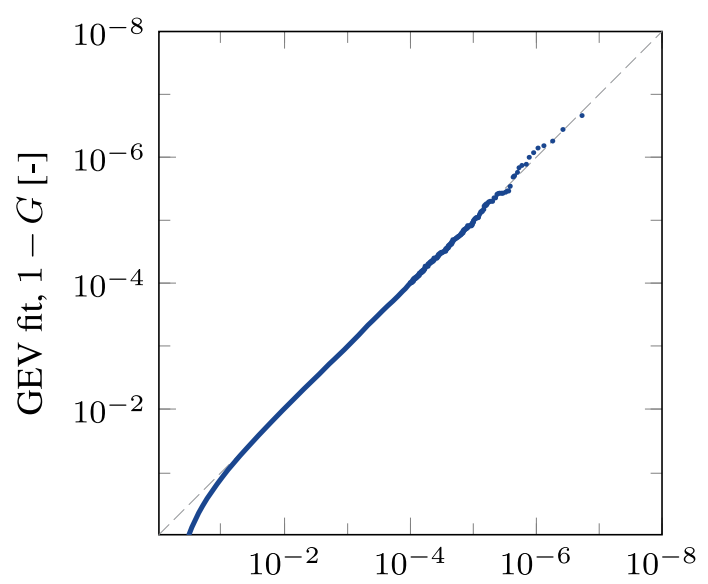

Empirical probability of exceedance, $1-\hat{F}[-]$

Figure 6. Q-Q plot, showing how well the empirical tail behavior matches a generalized extreme value distribution $(\mu=$ $78.2 \mathrm{MN} \times \mathrm{m} ; \sigma=2.05 \mathrm{MN} \times \mathrm{m} ; \xi=0.026)$.

cording to $f\left(\hat{M}_{50 \mathrm{yr}}\right)$ :

$$
\begin{aligned}
& \text { rank } \rightarrow \\
& M_{1,1} \leq M_{2,1} \leq \ldots \leq M_{N, 1} \rightarrow \hat{M}_{50 \mathrm{yr}, 1} \\
& M_{1,2} \leq M_{2,2} \leq \ldots \leq M_{N, 2} \rightarrow \hat{M}_{50 \mathrm{yr}, 2} \\
& \Xi \quad \vdots \quad \vdots \quad \vdots \\
& M_{1, j} \leq M_{2, j} \leq \ldots \leq M_{N, j} \rightarrow \hat{M}_{50 \mathrm{yr}, j} \\
& \vdots \quad \vdots \quad \vdots \\
& M_{1, k} \leq M_{2, k} \leq \ldots \leq M_{N, k} \rightarrow \hat{M}_{50 \mathrm{yr}, k} \\
& \downarrow \\
& f\left(\hat{M}_{50 \text { yr }}\right) \text {. }
\end{aligned}
$$

The medians and other quantiles are then estimated by sorting. For example, in case no. 4 (the crude Monte Carlo method with a GEV fitted above a threshold), the end result is the situation depicted in Fig. 7, which can be repeated for different sample sizes.

\section{Results}

Based on the load set and the extrapolation scheme, we can estimate how far a single 50-year load prediction would be off the true value. 
Table 1. Extrapolation cases.

\begin{tabular}{llll}
\hline & Sampling method (parent distribution) & $\begin{array}{l}\text { Distribution } \\
\text { fit }\end{array}$ & $\begin{array}{l}\text { Fitted only } \\
\text { above threshold }\end{array}$ \\
\hline (a) & Aggregation before fitting (Rayleigh) & Gumbel & No \\
(b) & Aggregation before fitting (Rayleigh) & Gumbel & Yes \\
(c) & Aggregation before fitting (Rayleigh) & GEV & No \\
(d) & Aggregation before fitting (Rayleigh) & GEV & Yes \\
(e) & Fitting before aggregation (Uniform) & Gumbel & No \\
(f) & Fitting before aggregation (Uniform) & Gumbel & Yes \\
(g) & Fitting before aggregation (Uniform) & GEV & No \\
(h) & Fitting before aggregation (Uniform) & GEV & Yes
\end{tabular}

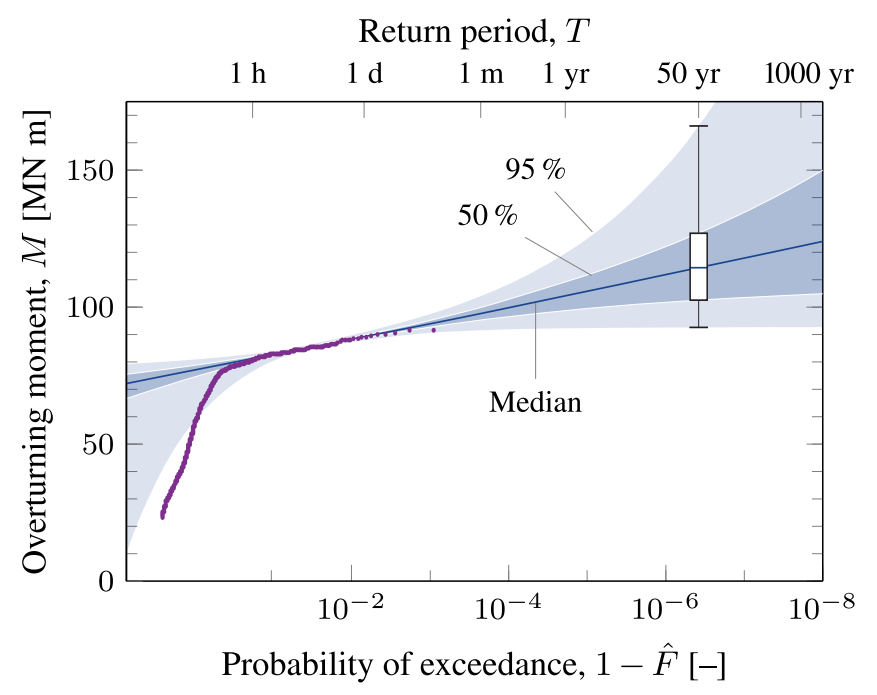

Figure 7. Return level plot of the tower base overturning moment, showing all the $k=10^{3} \mathrm{GEV}$ fits with a sample size of $N=10^{3}$. The dot markers belong to one of the $10^{3}$ samples.

\subsection{Uncertainty surrounding the 50-year overturning moment}

Figure 8 shows how the median and confidence intervals around the 50-year level vary as a function of sample size, $N$. Evidently, the larger the sample size, the smaller the error. In addition, there are some interesting differences between the eight approaches. Comparing the left column (panels a, c, $\mathrm{e}, \mathrm{g}$ ) to the right column (panels $\mathrm{b}, \mathrm{d}, \mathrm{f}, \mathrm{h}$ ), it seems that a threshold is indeed needed to establish a reliable fit of the distribution tail. Even when the data points are sampled from $1 \mathrm{~m} \mathrm{~s}^{-1}$ wide bins, the distributions are often bent and hardly match with any single distribution. The extreme case is when the full empirical load distribution of the aggregation-beforefitting approach (see, e.g., Fig. 2) is fitted to a Gumbel (panel a), producing errors well in excess of $+100 \%$. In the case of the GEV (panel c), the fit often takes on strongly negative values of $\xi$. This leads to a reversed Weibull distribution with an upper bound, which produces a negative bias.
The fitting-before-aggregation approach (panels e-h) tends to suffer from a positive bias. Likely, this is because most of the partial distributions have a slightly downwardly curved tail. Such a shape requires a large enough sample size to fully establish itself. Small sample sizes, on the other hand, tend to result in a fit with too large a slope that overpredicts the 50-year load.

The tail of the full data set has a slightly upwardly curved tail that matches a GEV distribution with a small positive $\xi$ best (see Figs. 2 and 6). However, the Gumbel distribution is clearly more forgiving at small sample sizes. A fixed $\xi=0$ has the advantage that the fit always stays close to the ideal value, which is especially helpful if the tail of the empirical distribution only contains a few data points.

In addition, the root-mean-squared (rms) error provides a single measure for the quality of the result:

$\varepsilon_{\mathrm{rms}}=\sqrt{\frac{1}{k} \sum_{j=1}^{k}\left(\hat{M}_{50 \mathrm{yr}, j}-M_{50 \mathrm{yr}}\right)^{2}}$,

where $M_{50 \mathrm{yr}}$ is the "true" 50-year level. As shown in Fig. 9, the aggregation-before-fitting approach with a Gumbel fitted above a threshold (b) produces the lowest rms error. Most of the other approaches show a clear improvement with increasing sample size. Ultimately, the rms error of (d) falls into the classic $1 / \sqrt{N}$ rule that is often found with Monte Carlo methods.

Of course, there are many other approaches to the extrapolation problem that lead to results of differing quality. In this paper, however, the focus is more on demonstrating how these errors affect a design process. Out of the eight approaches presented here, two are selected. The first is the aggregation-before-fitting approach with a GEV fit above a threshold (d), which has a relatively small bias but a large spread. The second is the fitting-before-aggregation approach with a Gumbel fitted above a threshold (f), which has a large bias but a smaller spread. 

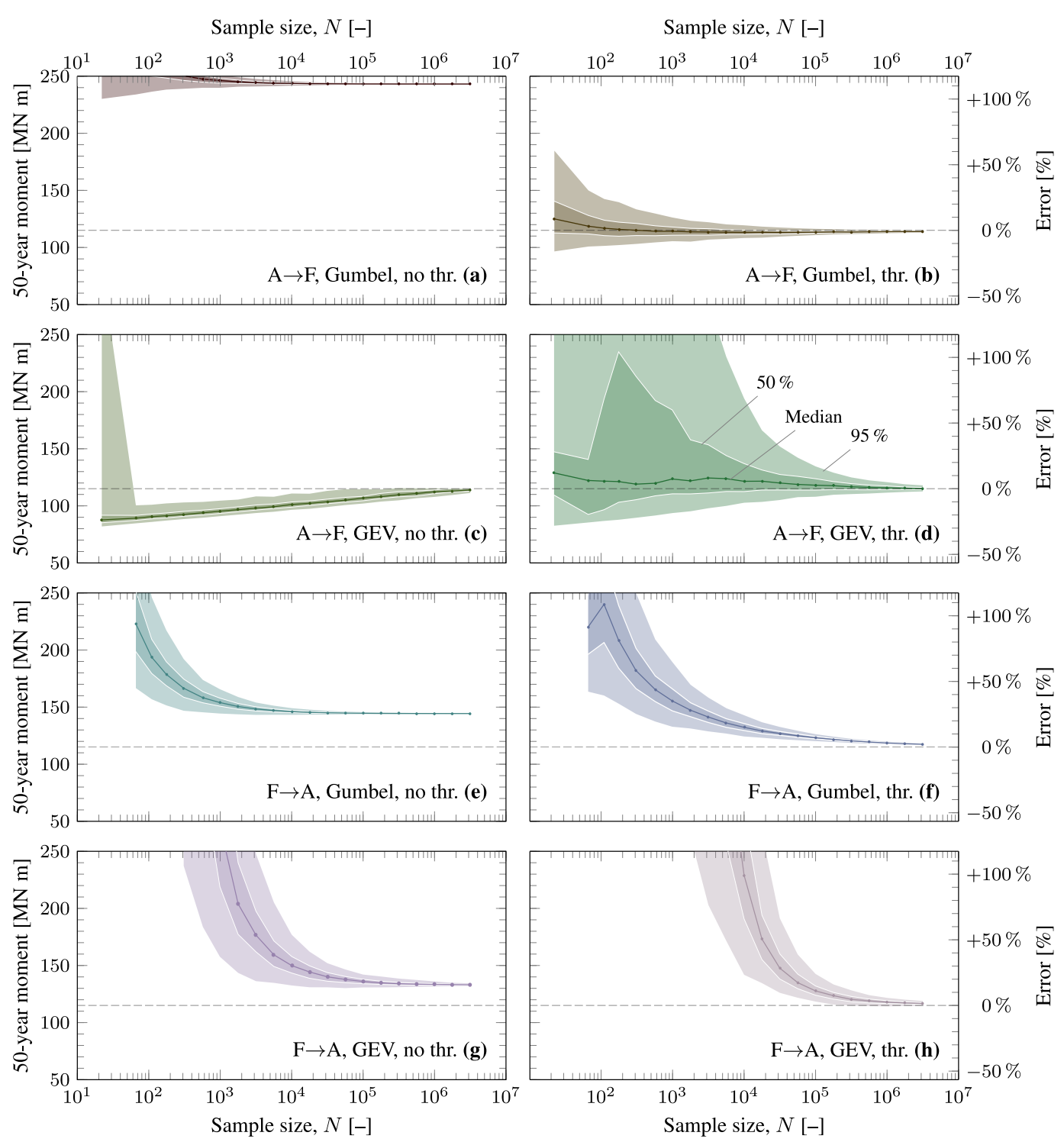

Figure 8. Error in the extrapolated 50-year overturning moment, as a function of sample size.

\subsection{Effect on decision making}

How this uncertainty affects the decision making process is demonstrated here with a very simple example, where the choice between two designs is based on a stress level. Say that a new concept is proposed that is an exact copy of the original NREL $5 \mathrm{MW}$ machine, but with a different wall thickness at the base of the tower. The second moment of area then changes according to

$I_{y y}=\frac{\pi}{4}\left[r^{4}-(r-t)^{4}\right]$,

where $r=3 \mathrm{~m}$ is the base radius and $t=35 \mathrm{~mm}$ is the original wall thickness (Jonkman et al., 2009). An extreme over- turning moment would cause a compressive stress of

$\sigma_{z}=\frac{M r}{I_{y y}}+\frac{m g}{A}$,

where $m g=6.82 \mathrm{MN}$ is the total weight of the wind turbine, and

$A=\pi\left[r^{2}-(r-t)^{2}\right]$

is the cross-sectional area of the tower base section.

The objective of the exercise is to find a new wall thickness to reduce the 50-year stress levels, i.e.,

$\sigma_{z, \text { new }}<\sigma_{z, \text { old }}$.

This might seem trivial at this point - any thicker wall is guaranteed to reduce the stresses - but the actual difficulty 


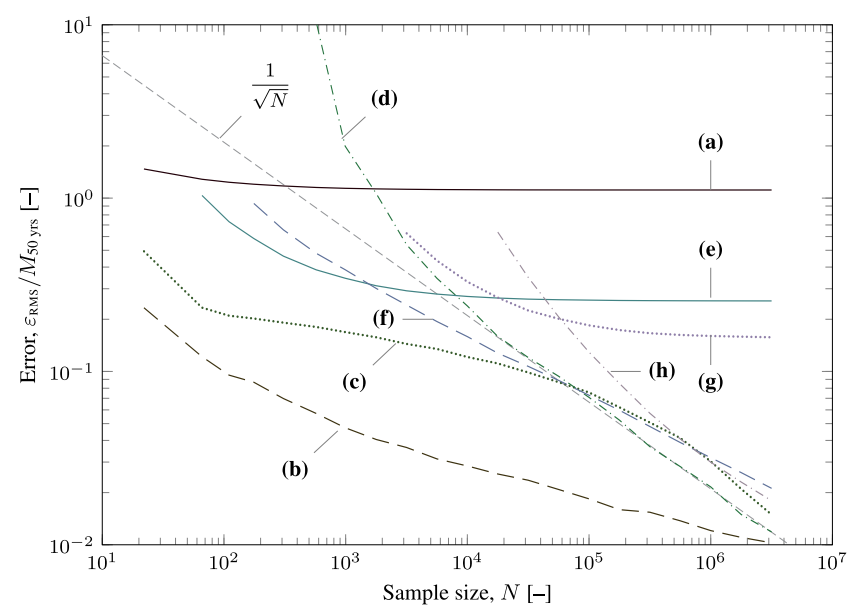

Figure 9. Root-mean-squared error in the extrapolated 50-year overturning moment, as a function of sample size.

is to determine the 50-year moment. Whereas the original design has already gone through an extensive load analysis from which the 50-year load level is known, any new concept has to go through this process again. ${ }^{2}$

Due to the uncertainty that surrounds this 50-year level, the new design can be falsely rejected or falsely accepted. Figure 10, for example, shows how often this happens when the load analysis is carried out with the aggregation-beforefitting approach and a sample size of $N=10^{3}$. When the wall thickness is reduced by $10 \%$ to $31.5 \mathrm{~mm}$, the new design will appear to have lower stresses in $18 \%$ of the cases (i.e., the false positives). On the other hand, even when the wall thickness is increased by $10 \%$ to $38.5 \mathrm{~mm}$, the new design has a $47 \%$ chance to still be rejected (i.e., the false negatives).

The closer a new design is to the original, the larger the required sample size (see Fig. 11). In the case of a large positive bias (see Fig. 12), a new concept is nearly always rejected, even if it has exactly the same thickness as the original.

Another case is a comparison between several concepts, where the 50-year stress levels contain the same degree of uncertainty. Five concepts, from 25 to $45 \mathrm{~mm}$ wall thickness, are ranked against each other, such that

$\sigma_{z, 1} \leq \sigma_{z, 2} \leq \sigma_{z, 3} \leq \sigma_{z, 4} \leq \sigma_{z, 5}$.

In the ideal case, the $45 \mathrm{~mm}$ wall thickness should end up at rank 1 , the $40 \mathrm{~mm}$ one at rank 2, etc. However, how often this ideal ranking happens in practice is shown in Figs. 13 and 14. This is where a small spread is preferred over a small bias. As long as the concepts are close to the same mean value, they can still be effectively compared. After $N=10^{4}$, the uncertainty is small enough for the order to be right roughly $100 \%$ of the time in the case of fitting before aggregation

\footnotetext{
${ }^{2}$ This may not be the case for something like a new wall thickness but rather for different control schemes or for rigorous changes to the blade design.
}

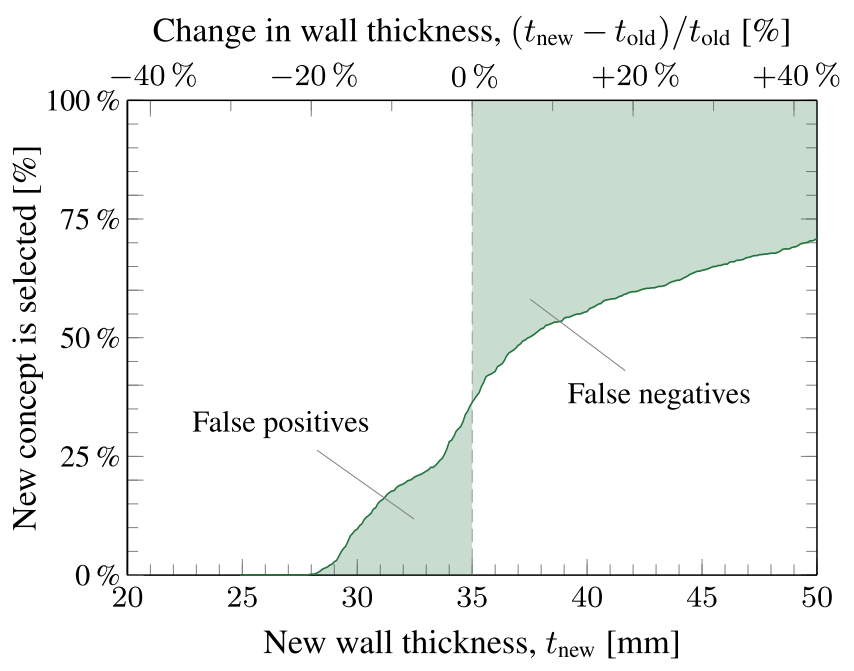

Figure 10. Outcome of a concept selection, where a new wall thickness is either accepted or rejected on the basis of a 50-year stress level using an aggregation-before-fitting approach with a GEV fitted above a threshold with a sample size of $N=10^{3}$.

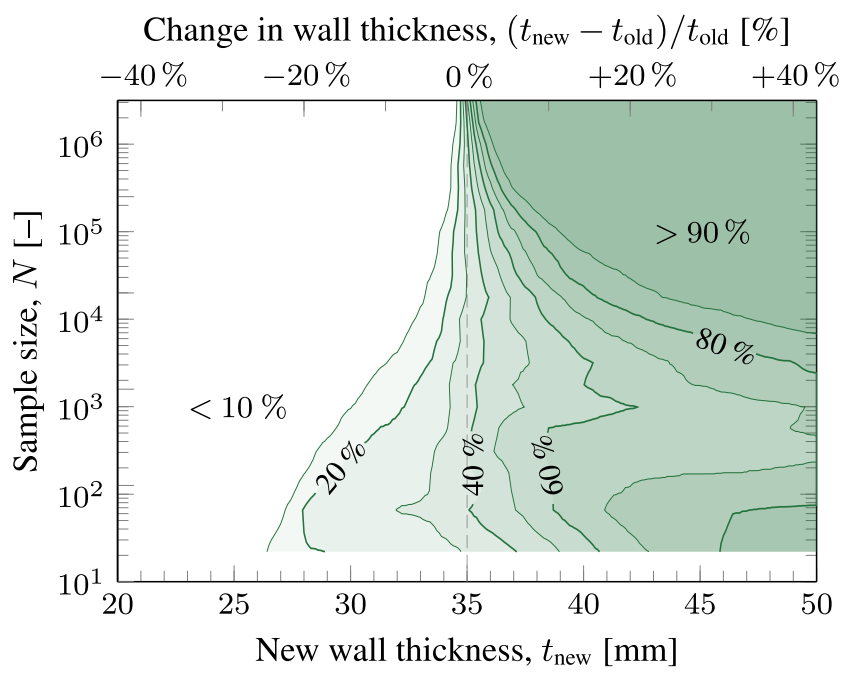

Figure 11. Relative number of times a new wall thickness is accepted on the basis of a 50-year stress level, predicted with an aggregation-before-fitting approach with a GEV fitted above a threshold.

(Fig. 14). For aggregation before fitting (Fig. 13), this is not until $N=3 \times 10^{5}$.

How often each rank is assigned to each concept is shown in Figs. 15 and 16. Clearly, the $45 \mathrm{~mm}$ wall thickness does not always appear the best and the $25 \mathrm{~mm}$ wall thickness does not always appear the worst. In fact, there are cases where the $45 \mathrm{~mm}$ wall thickness ends up being the worst of the five concepts (see Fig. 15 for $N=10^{2}$ ). 


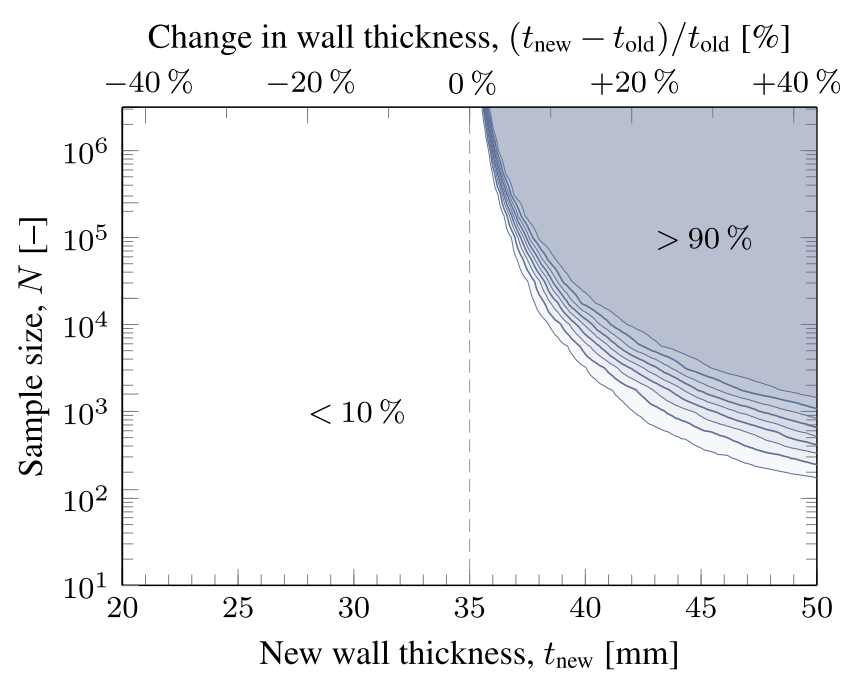

Figure 12. Relative number of times a new wall thickness is accepted on the basis of a 50-year stress level, predicted with a fittingbefore-aggregation approach with a Gumbel fitted above a threshold.

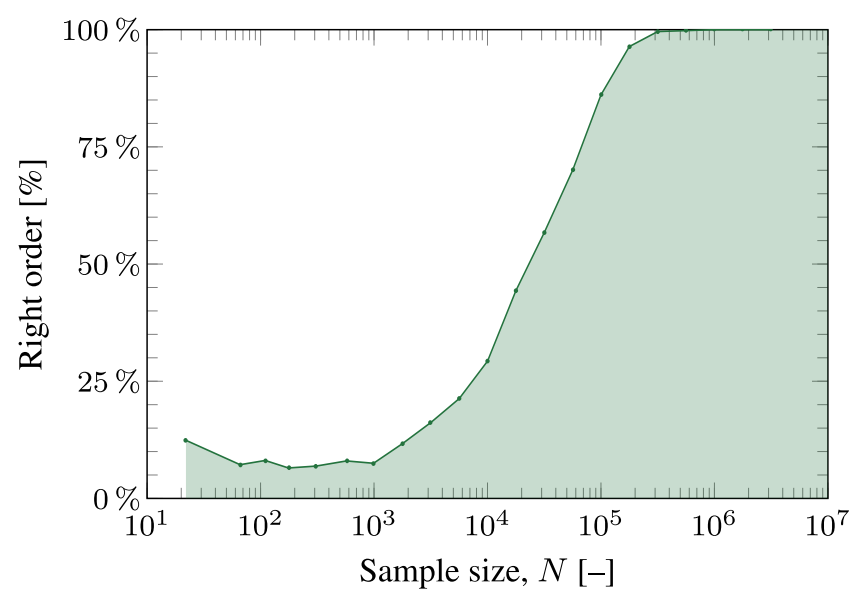

Figure 13. How often five concepts, ranging from 25 to $45 \mathrm{~mm}$ wall thickness, are ranked in the right order from lowest stress to highest using an aggregation-before-fitting approach with a GEV fitted above a threshold.

\section{Discussion}

The uncertainty around the 50-year level clearly has a very large impact on decision making. In this paper, we have focused on wall thickness in order to produce results that are counterintuitive. This is to demonstrate that extrapolated 50year values can be misleading and can easily trick the designer into making bad choices. In this case, the bad choices are obvious. However, they can be very difficult to spot in many other cases, for example, when choosing between different foundation types, tuning the gain settings for a controller, or even deciding whether DLC 1.1 is driving over other IEC load cases. The positive bias that is often present in

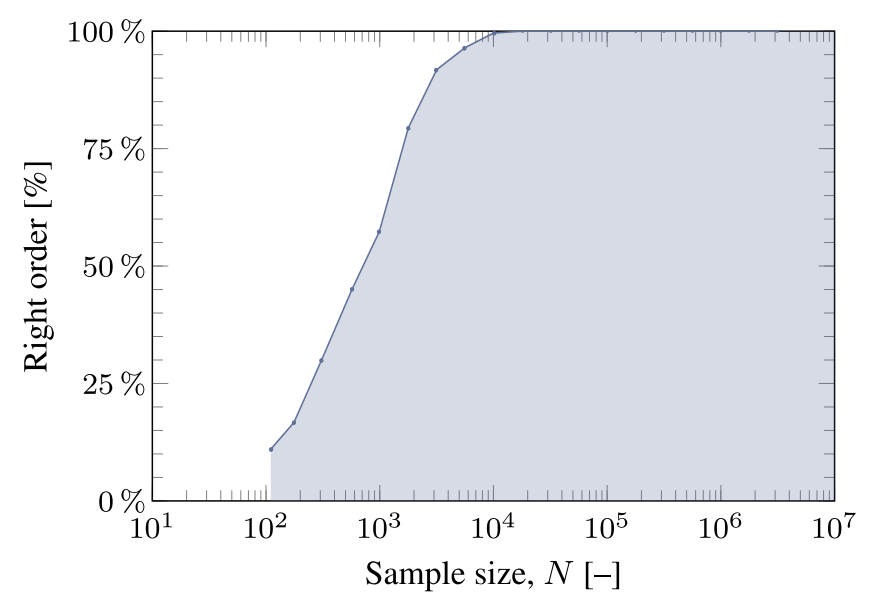

Figure 14. How often five concepts, ranging from 25 to $45 \mathrm{~mm}$ wall thickness, are ranked in the right order from lowest stress to highest using a fitting-before-aggregation approach with a Gumbel fitted above a threshold.

extreme load extrapolations (e.g., Barone et al., 2012b; van Eijk, 2016) makes it particularly difficult to prove that new designs are capable of reducing 50-year levels. However, it will take an immense computational effort to completely remove the uncertainty from the design process. It is therefore very important that the designer is skeptical enough of their own results.

Situations where good designs are wrongly discarded or where bad designs are wrongly accepted have a high chance of occurring when the sample sizes are small, especially during the initial design phases. In any case, we can conclude that the bare minimum of $300 \mathrm{~min}$ of time series, as prescribed in Appendix F of the standards (IEC, 2005), is not sufficient to produce any reasonable 50-year estimate (at least not when using one of the eight approaches in this paper). One should instead aim for sample sizes larger than $N=10^{4}$, and preferably larger than $N=10^{5}$. The effects of changes in wall thickness in the order of more than $10 \%$ are then easily recognizable.

The most obvious solution to reduce the uncertainty is to use high-performance computers in order to run extensive simulation campaigns (e.g., Barone et al., 2012a, b). An alternative remedy is to rely on importance sampling, which is a well-known variance reduction method that allows the user to allocate the computational resources for the most severe conditions (e.g., Bos et al., 2015; Bos and Veldkamp, 2016).

\section{Conclusions}

The goal of this paper was to demonstrate the effects of the uncertainty around extrapolated 50-year loads. It showed that, unless very large sample sizes are used, DLC 1.1 is a very unreliable measure for the performance of a design. This 

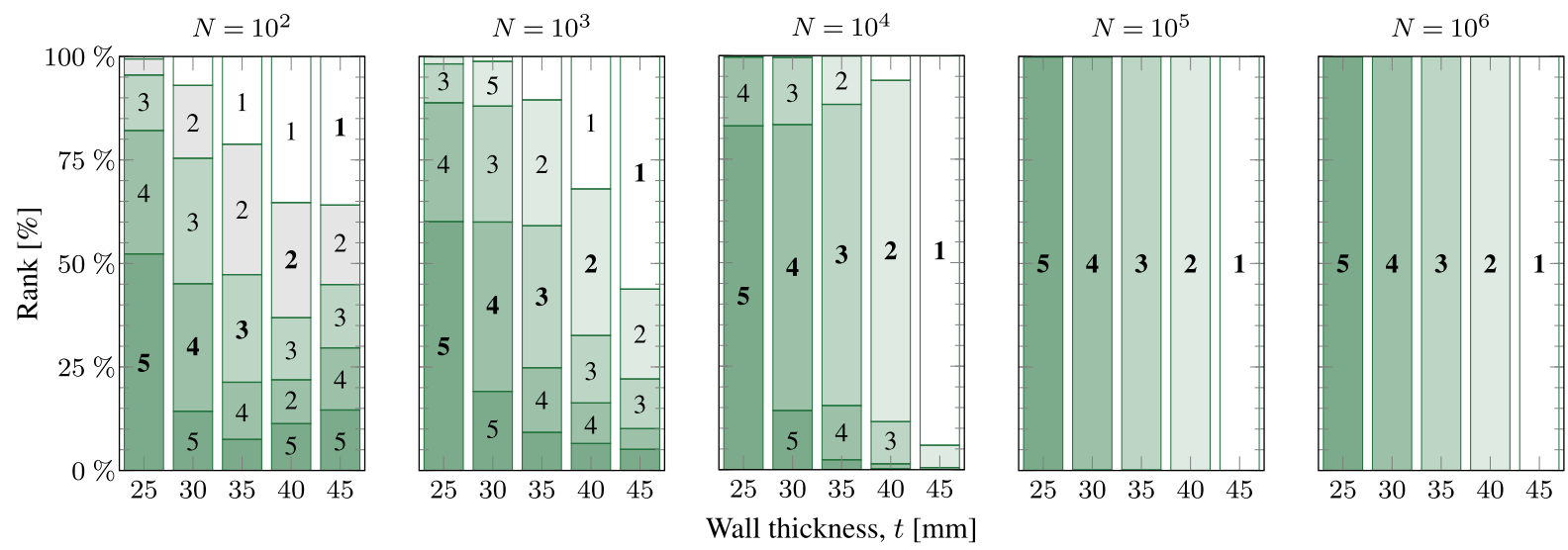

Figure 15. Ranking of five wall thicknesses on the basis of a 50-year stress level using an aggregation-before-fitting approach with a GEV fitted above a threshold with different sample sizes.
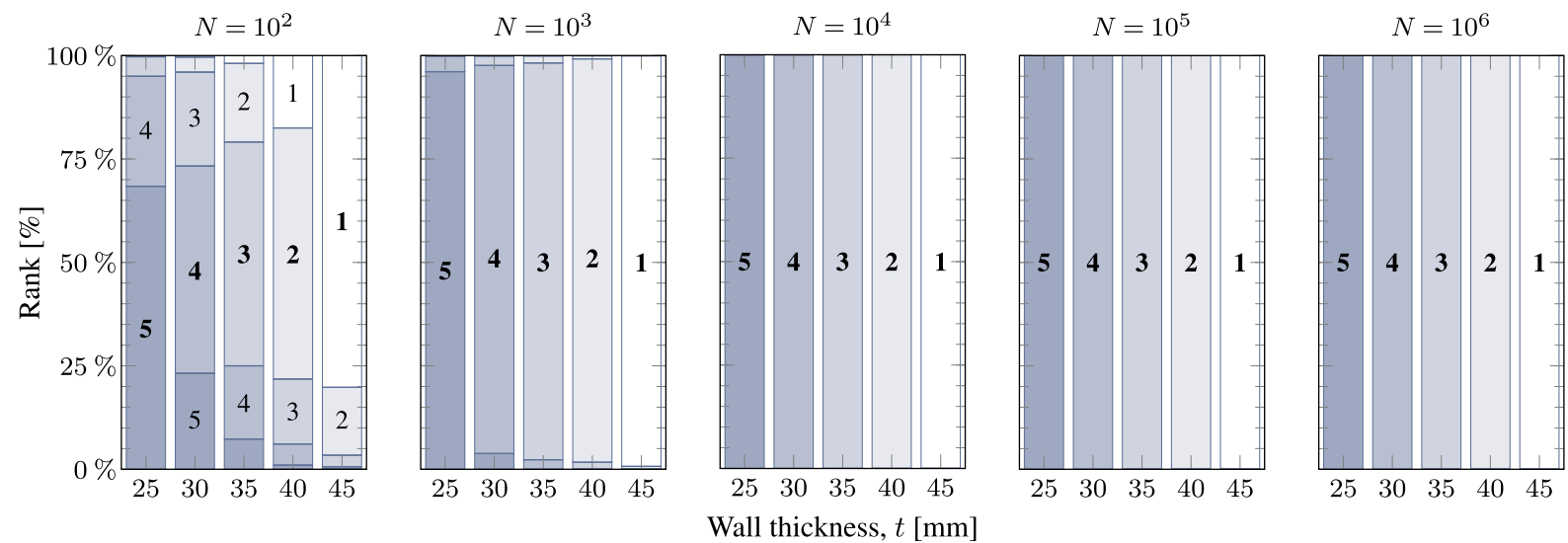

Figure 16. Ranking of five wall thicknesses on the basis of a 50-year stress level using a fitting-before-aggregation approach with a Gumbel fitted above a threshold with different sample sizes.

uncertainty has a pronounced effect on early phases of the design, when computational resources are often scarce.

One should always take into account that it is very timeconsuming to prove that concepts are able to reduce the 50year load, unless the design changes are very radical. In one example using an aggregation-before-fitting approach, where the bottom tower wall thickness of the NREL $5 \mathrm{MW}$ reference turbine was varied, a $10 \%$ increase in wall thickness was identified as a way to reduce the stress in only $53 \%$ of the cases with a sample size of $N=10^{3}$. In fact, more than $10^{5}$ simulations were required to decrease the probability of a false rejection to $10 \%$. Using a fitting-before-aggregation approach instead led to a strong positive bias and a rejection of the new concept in most cases.

These results show that a critical attitude is required when judging extrapolated extreme loads. When DLC 1.1 is not the design driver, it might be best to avoid it altogether in early phases of the design. Otherwise, using high-performance computing or importance sampling methods will be the best approach.
Data availability. The data set is from a third party (Sandia National Laboratories) and can be accessed at http://energy.sandia.gov/ ?page_id=13173.

Competing interests. The authors declare that they have no conflict of interest.

Acknowledgements. The authors would like to thank Sandia National Laboratories for making the wind turbine loads database available online.

Edited by: Michael Muskulus

Reviewed by: Nikolay Dimitrov and one anonymous referee

\section{References}

Agarwal, P. and Manuel, L.: Simulation of offshore wind turbine response for long-term extreme load prediction, Eng. Struct., 
31, 2236-2246, https://doi.org/10.1016/j.engstruct.2009.04.002, 2009.

Barone, M., Paquette, J., Resor, B., and Manuel, L.: Decades of wind turbine load simulation, 50th AIAA Aerosp. Sci. Meet. Incl. New Horizons Forum Aerosp. Expo., Nashville, TN, United States, 9-12 January 2012, https://doi.org/10.2514/6.2012-1288, 2012a.

Barone, M., Paquette, J., Resor, B., Manuel, L., and Nguyen, H.: Simulating the entire life of an offshore wind turbine, EWEA Annual Event, Copenhagen, Denmark, 16-19 April, 2012b.

Bos, R. and Veldkamp, H. F.: A method to find the 50-year extreme load during production, J. Phys. Conf. Ser., 753, 42021, https://doi.org/10.1088/1742-6596/753/4/042021, 2016.

Bos, R., Bierbooms, W. A. A. M., and van Bussel, G. J. W.: Importance sampling of severe wind gusts, 11 th $\mathrm{PhD}$ Semin. Wind Energy Eur., Stuttgart, Germany, 23-25 September 2015.

Fogle, J., Agarwal, P., and Manuel, L.: Towards an improved understanding of statistical extrapolation for wind turbine extreme loads, Wind Energy, 11, 613-635, https://doi.org/10.1002/we.303, 2008.

Freudenreich, K. and Argyriadis, K.: The load level of modern wind turbines according to IEC 61400-1, J. Phys. Conf. Ser., 75, 12075, https://doi.org/10.1088/1742-6596/75/1/012075, 2007.

Genz, R., Nielsen, K. B., and Madsen, P. H.: An investigation of load extrapolation according to IEC 61400-1, 3rd Edn., Eur. Wind Energy Conf., Athens, Greece, 27 February-2 March 2006.

IEC: 61400-1 Wind turbines - Part 1: Design requirements, 3rd edn., International Electrotechnical Commission, Geneva, Switzerland, 2005.

Jonkman, J. M., Butterfield, S., Musial, W., and Scott, G.: Definition of a 5-MW reference wind turbine for offshore system development, Technical Report NREL/TP-500-38060, National Renewable Energy Laboratory, Golden, CO, USA, 2009.
Lott, S. and Cheng, P.-W.: Load extrapolations based on measurements from an offshore wind turbine at alpha ventus, J. Phys. Conf. Ser., 753, 072004, https://doi.org/10.1088/17426596/753/7/072004, 2016.

Moriarty, P. J., Holley, W. E., and Butterfield, S. P.: Extrapolation of extreme and fatigue loads using probabilistic methods, Tech. rep. NREL/TP-500-34421, National Renewable Energy Laboratory, Golden, CO, USA, 2004.

Natarajan, A. and Holley, W. E.: Statistical extreme load extrapolation with quadratic distortions for wind turbines, J. Sol. Energy Eng., 130, 031017, https://doi.org/10.1115/1.2931513, 2008.

Pandey, M. D. and Sutherland, H. J.: Probabilistic analysis of LIST data for the estimation of extreme design loads for wind turbine components, J. Sol. Energy. Eng., 125, 531-540, https://doi.org/10.1115/1.1626128, 2003.

Peeringa, J.: Comparison of extreme load extrapolations using measured and calculated loads of a MW wind turbine, Eur. Wind Energy Conf., Marseille, France, 16-19 March 2009.

Ragan, P. and Manuel, L.: Statistical extrapolation methods for estimating wind turbine extreme loads, 45th Aerosp. Sci. Meet. Exhib., Reno, NV, USA, 8-11 January 2007.

van Eijk, S. F.: Wind turbine load extrapolation, Master's thesis, Delft University of Technology, 2016. 